\title{
PLANETARY ROVER NAVIGATION: IMPROVING VISUAL ODOMETRY VIA ADDITIONAL IMAGES AND MULTISENSOR FUSION
}

\section{G. Casalino, E. Zereik, E. Simetti, A. Turetta, S. Torelli, and A. Sperindé}

\author{
Department of Communication, Computer and System Science \\ University of Genoa \\ 13 Via Opera Pia, Genoa 16145, Italy
}

\begin{abstract}
Visual odometry (VO) is very important for a mobile robot, above all in a planetary scenario, to accurately estimate the rover occurred motion. The present work deals with the possibility to improve a previously developed VO technique by means of additional image processing, together with suitable mechanisms such as the classical Extended/Iterated Kalman Filtering and also Sequence Estimators. The possible employment of both techniques is then addressed and, consequently, a better behaving integration scheme is proposed. Moreover, the eventuality of exploiting other localization sensors is also investigated, leading to a final multisensor scheme.
\end{abstract}

\section{INTRODUCTION}

This work preliminarily quotes an VO technique, previously developed at GRAAL Lab, for the estimation of the relative poses assumed by a moving vehicle at discrete instants of time (for details about the developed VO algorithm, refer to [1-4]. Then, it approaches the problem of improving the VO-provided estimation sequences: the suggested method reasonably plans to add to two successive acquisitions a further one linking the two; moreover, suitable mechanisms allowing a sensible reduction of the drifting errors, generally occurring when the absolute poses are deduced via summation of the relative ones, can be devised. More specifically, to the aim of such drifting reduction, state estimation techniques (Extended Kalman Filtering (EKF) and/or Iterated Kalman Filtering $(\mathrm{IKF}))$ are formerly suggested. Then, as an alternative to state estimation, the better technique of sequence estimation, even if computationally a little more 
cumbersome, is also suggested and tailored to the problem. Moreover, the possibility of suitably integrating the two techniques (state estimation and sequence estimation) into an overall scheme, better behaving than when using the same technique separately, is analyzed and then suggested to be adopted. Finally the possible integration of other sensors (namely, Inertial Measurement Units (IMU), and Wheel Odometry (WO) systems) is also briefly investigated within the definition of an overall resulting, further improved, multisensor integration scheme.

\section{VISUAL ODOMETRY BASED ADDITIONAL IMAGE PROCESSING}

The VO technique, once per-se considered, can be seen as nothing more than a sensing system capable of progressively constructing, generally, in the form of assumed independent estimates, the chained set of frames of Fig. 1; each frame is associated to a point located on a realization of a path traveled by a vehicle, without actually keeping into account anything about its specific motion characteristics. In Fig. 1, each couple ${ }^{i-1} r_{i}$ and ${ }^{i-1} \theta_{i}$ (relative position and orientation) represents the true realized motion, completely defining the relative position of frame $\langle i\rangle$ with respect to its antecedent $\langle i-1\rangle$; concerning this, the VO technique progressively provides their estimates, that is, the couple of quantities ${ }^{i-1} \hat{r}_{i}$ and ${ }^{i-1} \hat{\theta}_{i}$, characterized by the associated variances ${ }^{i-1} \Sigma_{i}$ and ${ }^{i-1} \Theta_{i}$ respectively, also provided by the VO technique itself. Note that just for sake of simplicity, it has been assumed that the two estimates ${ }^{i-1} \hat{r}_{i}$ and ${ }^{i-1} \hat{\theta}_{i}$ are uncorrelated among them (no cross-variance terms have been actually evidenced). As it will be shown below, this assumption will, however, generally reveal to stand without loss of generality. Moreover, also note that, due to the

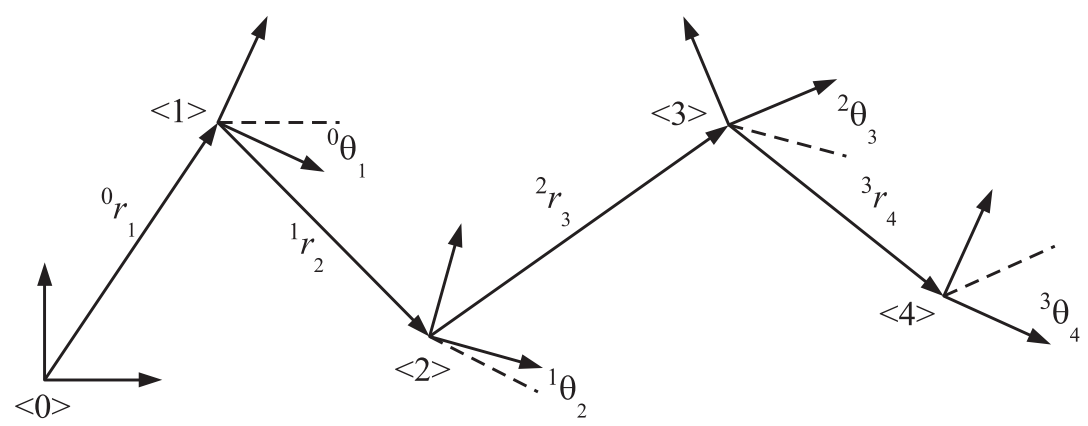

Figure 1 Sequence of positions-orientations attained by the vehicle 
specific way the VO actually works, each couple of estimates ${ }^{i-1} \hat{r}_{i}$ and ${ }^{i-1} \hat{\theta}_{i}$ can be considered as independent from all the others. This fact allows to write the following relationships:

$$
\begin{aligned}
& { }^{i-1} r_{i}={ }^{i-1} \hat{r}_{i}+{ }^{i-1} \varepsilon_{i} ; \quad{ }^{i-1} \varepsilon_{i}=N\left({ }^{i-1} \varepsilon_{i}, 0,{ }^{i-1} \Sigma_{i}\right) \\
& { }^{i-1} \theta_{i}={ }^{i-1} \hat{\theta}_{i}+{ }^{i-1} \eta_{i} ; \quad{ }^{i-1} \eta_{i}=N\left({ }^{i-1} \eta_{i}, 0,{ }^{i-1} \Theta_{i}\right)
\end{aligned}
$$

with $\varepsilon_{i}$ and $\eta_{i}$ independent (among them as above assumed) white sequences and reasonably considered Gaussian with zero mean as a working assumption. Keeping into account the above considerations, it can be immediately seen how the VO technique can provide estimates of the absolute positions and orientations progressively attained by the vehicle only in the following form (i. e., the classical product of planar transformation matrices of each frame with respect to its antecedent)

$$
{ }^{0} \hat{T}_{i}={ }^{0} \hat{T}_{1}{ }^{1} \hat{T}_{2} \ldots{ }^{i-1} \hat{T}_{i}
$$

with

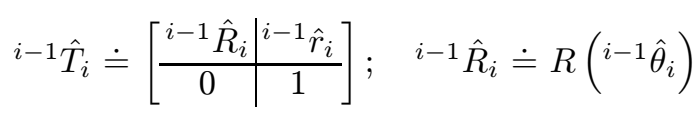

which is clearly prone to an error progressively increasing with the number of stages. As a matter of fact, such evidenced drawback simply arises from the fact that the VO algorithm, as above applied, leads per-se to a linear open chain of frames, with independent positioning of each one with respect to its antecedent, without any further constraint. Hence, in the following, it will be primarily investigated about the possibility of improving this technique, by forcing it to provide further measurements, introducing some (probabilistic) constraints among the basic chained frames, in order to improve the vehicle absolute localization. To this aim, a feasible possibility is that of adding to the basic measurements the estimation of frame $\langle i\rangle$ position also with respect to frame $\langle i-2\rangle$; in this

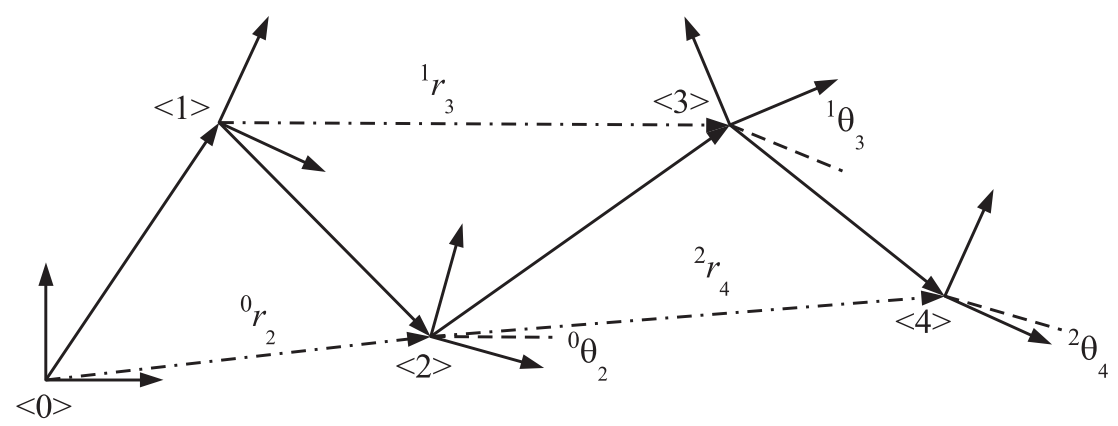

Figure 2 Adding extra image tracking to the basic ones 
way, it is possible to refer to the progressively growing graph of frames (now including closed loops connections) reported in Fig. 2, where only the additional estimate true values are indicated. As it can be easily seen, since the following relationships among the true values always apply (binding together two successive relative frame positions and attitudes)

$$
\begin{aligned}
{ }^{i-2} r_{i} & ={ }^{i-2} r_{i-1}+R\left({ }^{i-2} \theta_{i-1}\right){ }^{i-1} r_{i} ; \\
{ }^{i-2} \theta_{i} & ={ }^{i-2} \theta_{i-1}+{ }^{i-1} \theta_{i},
\end{aligned}
$$

it follows that the new measurements ${ }^{i-2} \hat{r}_{i}$ and ${ }^{i-2} \hat{\theta}_{i}$ can be exploited for estimation improvement purposes. Note, however, that the assumption about the availability of the additional measurements requires that the stereocamera can recognize, within the current frame, not only a sufficient number of features belonging to the image just before this last, but also a sufficient number of those belonging to the two-step delayed one; this requirement, even if a little strict, seems reasonable to be implemented.

\section{STATE ESTIMATION}

With the introduction of the assumed additional measurements, let first observe how the sequence of acquisitions consequently evolves during time, with the aid of the scheme of Fig. 3. Then let decompose the process into stages corresponding, each one, to when the interlaced additional acquisition ${ }^{i-2} \hat{r}_{i},{ }^{i-2} \hat{\theta}_{i}$ has been completed, as indicated in Fig. 3; then observe how the so-resulting process can be modeled via the use of the following couple of separate, interacting, statespace models:

- basic relative-orientation dynamic

$$
\begin{aligned}
{\left[\begin{array}{c}
{ }^{i-1} \theta_{i} \\
{ }^{i-2} \theta_{i-1}
\end{array}\right] } & =\left[\begin{array}{ll}
0 & 0 \\
1 & 0
\end{array}\right]\left[\begin{array}{c}
{ }^{i-2} \theta_{i-1} \\
{ }^{i-3} \theta_{i-2}
\end{array}\right]+\left[\begin{array}{l}
1 \\
0
\end{array}\right]{ }^{i-1} \hat{\theta}_{i}+\left[\begin{array}{l}
1 \\
0
\end{array}\right]{ }^{i-1} \eta_{i} ; \\
x_{i}^{1} & =A_{1} x_{i-1}^{1}+B_{1} u_{i}^{1}+B_{1} \xi_{i}^{1} ;
\end{aligned}
$$

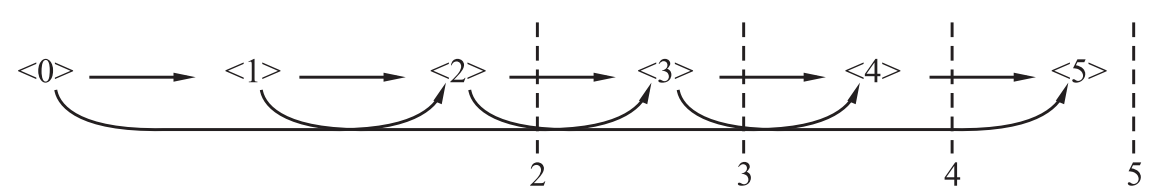

Figure 3 The considered sequence of stages 


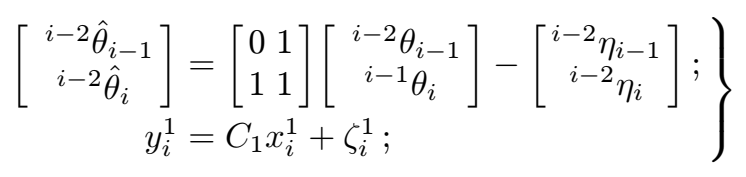

- basic relative-position dynamic

$$
\begin{aligned}
& \left.\begin{array}{rl}
{\left[\begin{array}{c}
{ }^{i-1} r_{i} \\
{ }^{i-2} r_{i-1}
\end{array}\right]} & =\left[\begin{array}{ll}
0 & 0 \\
I & 0
\end{array}\right]\left[\begin{array}{c}
{ }^{i-2} r_{i-1} \\
{ }^{i-3} r_{i-2}
\end{array}\right]+\left[\begin{array}{l}
I \\
0
\end{array}\right]{ }^{i-1} \hat{r}_{i}+\left[\begin{array}{l}
I \\
0
\end{array}\right]{ }^{i-1} \varepsilon_{i} ; \\
x_{i}^{2} & =A_{2} x_{i-1}^{2}+B_{2} u_{i}^{2}+B_{2} \xi_{i}^{2}
\end{array}\right\}
\end{aligned}
$$

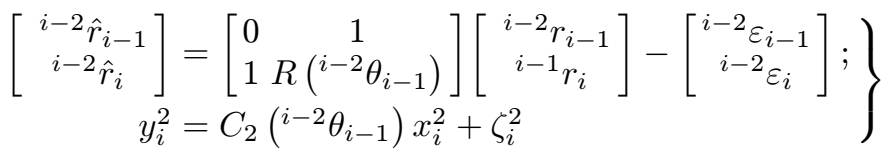

where also the compact standard notation has been reported (with obvious meaning of the introduced symbols). In particular, note how the interaction between the two systems is solely represented by the influence that the component ${ }^{i-2} \theta_{i-1}$ of the first system state exerts on rotation matrix $R\left({ }^{i-2} \theta_{i-1}\right)$ which, in turn, appears within the second output of the second system. This is actually the sole cause of nonlinearity of the resulting overall system that, otherwise, would have been fully linear and stationary. The signal flow scheme reported in Fig. 4 clearly shows the indicated interaction occurring between the two systems.

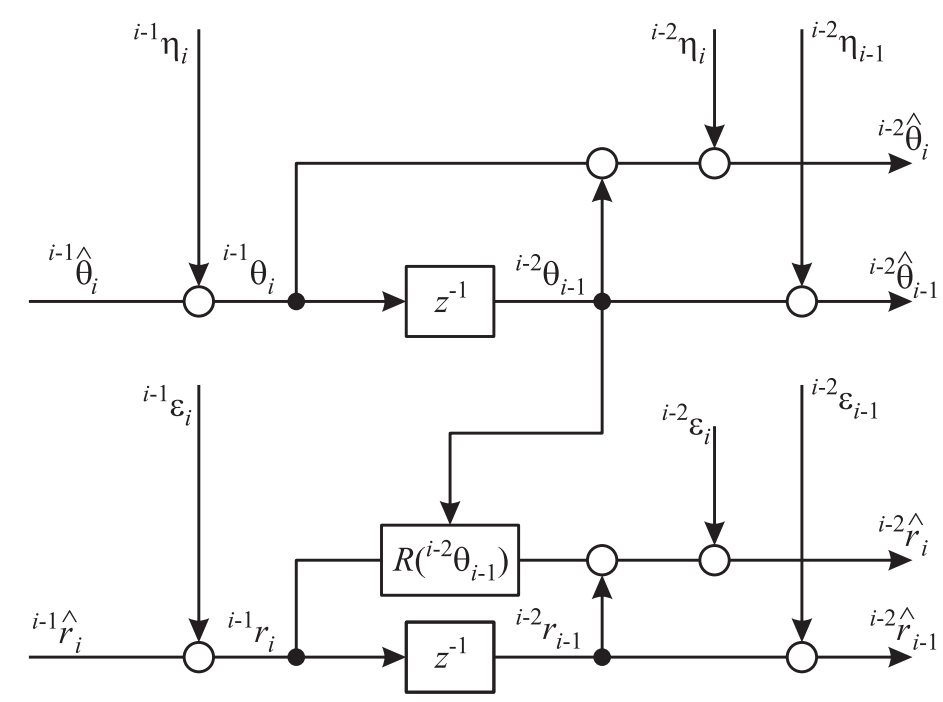

Figure 4 Block diagram of the considered state space model 


\subsection{Extended Kalman Filtering}

The above introduced overall state space system (i.e., the aggregation of the two) results into a linear time-invariant one as regards its dynamic part, while it is partially nonlinear in its overall output vector, as previously evidenced. As a consequence, the overall system evolution appears to be very suitable (i. e., certainly, more suitable than systems exhibiting nonlinearities also in their dynamic part) to be recursively estimated via the well known and celebrated EKF technique [5]. More precisely, one can formally aggregate the two systems into the following overall one (again, with an obvious meaning for the introduced symbols):

$$
\left.\begin{array}{c}
x_{i}=A x_{i-1}+B u_{i}+B \xi_{i} ; \quad Q_{i} \doteq \operatorname{cov}\left(\xi_{i}\right)=B \operatorname{diag}\left({ }^{i-1} \Theta_{i},{ }^{i-1} \Sigma_{i}\right) \\
y_{i}=C\left({ }^{i-2} \Theta_{i-1}\right) x_{i}+\zeta_{i} ; \\
S_{i} \doteq \operatorname{cov}\left(\zeta_{i}\right)=B \operatorname{diag}\left({ }^{i-2} \Theta_{i-1},{ }^{i-2} \Theta_{i},{ }^{i-2} \Sigma_{i-1},{ }^{i-2} \Sigma_{i}\right) .
\end{array}\right\}
$$

Then, by keeping into account the resulting linearity of the overall dynamic part and the kind of structured state dependency exhibited by the output matrix $C\left({ }^{i-2} \Theta_{i-1}\right)$, it is not difficult (even if a little tedious) to verify that the EKF associated to the above system actually takes on the form:

$$
\begin{aligned}
\widehat{x}_{i / i-1} & =A \widehat{x}_{i-1 / i-1}+B u_{i} \\
\widehat{x}_{i / i} & =\widehat{x}_{i / i-1}+K_{i}\left[y_{i}-C\left({ }^{i-2} \widehat{\Theta}_{i-1 / i-1}\right) \widehat{x}_{i / i-1}\right] ; \\
P_{i / i-1} & =A P_{i-1 / i-1} A^{\mathrm{T}}+B Q_{i} B^{\mathrm{T}} \\
P_{i / i} & =\left(I-K_{i} H_{i}\right) P_{i / i-1}
\end{aligned}
$$

with

$$
\begin{aligned}
& K_{i}=P_{i / i-1} H_{i}^{\mathrm{T}}\left(H_{i} P_{i / i-1} H_{i}^{\mathrm{T}}+S_{i}\right)^{-1} ; \\
& H_{i} \doteq C\left({ }^{i-2} \widehat{\Theta}_{i-1 / i-1}\right)+C^{\prime}\left({ }^{i-2} \widehat{\Theta}_{i-1 / i-1},{ }^{i-1} \widehat{r}_{i / i-1}\right)
\end{aligned}
$$

and where $C^{\prime}\left({ }^{i-2} \widehat{\Theta}_{i-1 / i-1},{ }^{i-1} \widehat{r}_{i / i-1}\right)$ simply has the "almost empty" form:

$$
\begin{aligned}
C^{\prime}\left({ }^{i-2} \widehat{\Theta}_{i-1 / i-1},{ }^{i-1} \widehat{r}_{i / i-1}\right) & {\left[\begin{array}{cccc}
0 & 0 & 0 & 0 \\
0 & 0 & 0 & 0 \\
0 & 0 \times 1 & 0_{2 \times 1} & 0_{2 \times 1} \\
0_{2 \times 1} & R^{\prime}\left({ }^{i-2} \widehat{\theta}_{i-1 / i-1}\right)^{i-1} \widehat{r}_{i / i-1} & 0_{2 \times 1} & 0_{2 \times 1}
\end{array}\right], }
\end{aligned}
$$


that is, a $6 \times 6$ square matrix only having nonzero elements in its second column. Moreover, within the indicated expression for such nonzero terms, one has:

$$
R^{\prime}\left({ }^{i-2} \widehat{\theta}_{i-1 / i-1}\right) \doteq\left[\begin{array}{cc}
-\sin { }^{i-2} \widehat{\theta}_{i-1 / i-1} & -\cos { }^{i-2} \widehat{\theta}_{i-1 / i-1} \\
\cos { }^{i-2} \widehat{\theta}_{i-1 / i-1} & -\sin { }^{i-2} \widehat{\theta}_{i-1 / i-1}
\end{array}\right]
$$

simply corresponding to the derivative with respect to $\theta$ of rotation matrix $R(\theta)$ evaluated for ${ }^{i-2} \widehat{\theta}_{i-1 / i-1}$. This section may be now concluded by explicitly noting how the presence of the nonzero terms in Eq. (13) is actually the sole responsible for having the filter of full-order 6 . In fact, as it can be easily verified, in case the nonzero terms in Eq. (13) were neglected, the overall filter would automatically reduce to two separate filters: the first one applied to system (3) and independently estimating the orientations and the second one applied to system (4), estimating the position only (even if this is done by also acquiring the orientation estimates from the first filter in order to evaluate — via direct insertion of such orientation estimates - the posture dependent output matrix appearing Eq. (4)). Obviously enough, though neglecting such terms in Eq. (13) certainly reduces the filter performances, the simplification gained in its implementation might, however, compensate for such performance reduction, provided that it turns out to be acceptable. Certainly enough, some simulation experiments should be performed to confirm (or not) such a conjecture.

\subsection{Iterated Kalman Filtering}

The IKF $[5,7]$ is a quasi-optimal nonlinear filter, which can actually be interpreted as an improvement introduced in the above seen EKF (that can, in turn, be seen as a simplification of IKF). There can be introduced the filter starting from the main part of the well-known Chapman-Kolmogorov general relationship, stating that having a dynamic system in the generic form

$$
x_{i}=f\left(x_{i-1}, u_{i}\right)+\xi_{i} ; \quad y_{i}=g\left(x_{i}\right)+\zeta_{i}
$$

together with the progressive collection of its output and input measurements

$$
I_{i}=\left\{\left(y_{k}, u_{k}\right) ; k=1,2, \ldots, i\right\},
$$

the posterior probability density function (p.d.f.) $p\left(x_{i} / I_{i}\right)$ can be expressed as

$$
p\left(\frac{x_{i}}{I_{i}}\right)=\frac{1}{M_{i}} p\left(\frac{y_{i}}{x_{i}}\right) p\left(\frac{x_{i}}{I_{i-1}}, u_{i}\right) .
$$

In the above relationship, the term $p\left(x_{i} / I_{i-1}, u_{i}\right)$ is the so-called prior p.d.f., that is the predicted distribution of $x_{i}$ before acquiring the current measurement set 
$y_{i} ; p\left(y_{i} / x_{i}\right)$ is the so-called Likelihood p.d.f., upgrading the prior p.d.f. into the posterior one, once the measurement $y_{i}$ is acquired. Finally, the quantity $M_{i}$ is simply the normalizing factor allowing the overall right-hand side to exhibit a unitary integral within the whole space. As it is well known, upgrading the posterior $p\left(x_{i} / I_{i}\right)$ starting from the previous one $p\left(x_{i-1} / I_{i-1}\right)$ represents the conceptual framework on the basis of which any state estimation technique (even if suboptimal) must be constructed. To this respect, the Chapman-Kolmogorov relationship in Eq. (14) clearly shows how the process should consequently adhere to the following logic:

$$
\begin{aligned}
p\left(\frac{x_{i-1}}{I_{i-1}}\right) \rightarrow p\left(\frac{x_{i}}{I_{i-1}}, u_{i}\right) \rightarrow & \bullet \rightarrow p\left(x_{i} / I_{i}\right) . \\
& \uparrow \\
& p\left(\frac{y_{i}}{x_{i}}\right)
\end{aligned}
$$

Within the above logic process and in case of nonlinear dynamic, the step in the left, i. e., the evaluation of the prior probability $p\left(x_{i} / I_{i-1}, u_{i}\right)$, is generally the most difficult one, while it results very simple in case of linear dynamics as in Eq. (9), Gaussian noises, and also Gaussian previous prior $p\left(x_{i-1} / I_{i-1}\right)$. More precisely provided that

$$
p\left(\frac{x_{i-1}}{I_{i-1}}\right)=N\left(x_{i-1}, \widehat{x}_{i-1 / i-1}, P_{i-1 / i-1}\right),
$$

it is well known that with a linear dynamic and white Gaussian noise, as it actually is for the considered case, one has

$$
p\left(\frac{x_{i}}{I_{i-1}}, u_{i}\right)=N\left(x_{i}, \widehat{x}_{i / i-1}, P_{i / i-1}\right)
$$

with $\widehat{x}_{i / i-1}$ and $P_{i / i-1}$ just provided by relationships in Eqs. (11) and (12), respectively, here repeated and renumbered for the sake of convenience and from now on reconsidered with respect to the considered case:

$$
\widehat{x}_{i / i-1}=A \widehat{x}_{i-1 / i-1}+B u_{i} ; \quad P_{i / i-1}=A P_{i-1 / i-1} A^{\mathrm{T}}+B Q_{i} B^{\mathrm{T}} .
$$

The evaluation of the Likelihood p.d.f. is generally much easier than the prior one (in particular, for the considered case, it is actually direct). In fact, from Eq. (10), one immediately has:

$$
p\left(\frac{y_{i}}{x_{i}}\right)=N\left[y_{i}, C\left({ }^{i-2} \theta_{i-1}\right) x_{i}, S_{i}\right],
$$

thus leading, together with Eq. (15) and the general Eq. (14), to the following expression for the posterior p.d.f.:

$$
p\left(\frac{x_{i}}{I_{i}}\right)=\frac{1}{M_{i}} N\left[y_{i}, C\left({ }^{i-2} \theta_{i-1}\right) x_{i}, S_{i}\right] N\left(x_{i}, \widehat{x}_{i / i-1}, P_{i / i-1}\right)
$$


Note, however, that, in spite of its first appearance at first glance, the above resulting posterior p.d.f. is non-Gaussian due to the nonlinear dependence of matrix $C\left({ }^{i-2} \theta_{i-1}\right)$ from the second component ${ }^{i-2} \theta_{i-1}$ of the whole state $x_{i}$; this means that any successive propagation of the posterior p.d.f., in the same form leading to Eq. (16), can be done only at the expense of suitably approximating $p\left(x_{i} / I_{i}\right)$ at each stage with a Gaussian p.d.f., before the next stage $i+1$ is executed. Such an approximation leads to suboptimal estimation techniques, at least for systems exhibiting nonlinearities in the output measurements only (like the one under consideration); these methods, anyway, turn out to be quite efficient, even if compared to more sophisticated ones (like, for instance, the recently introduced Monte Carlo based recursive techniques, generally leading to the Particle Filters - see [6-8] — whose implementation is generally much more cumbersome). Particle filtering techniques have been proposed for explicitly dealing with systems which are mainly nonlinear in their dynamic part, as in Eq. (5). They efficiently use real-time Monte Carlo methods to propagate the prior p.d.f., strongly influenced by the nonlinearity in the dynamic part (which, however, is not the considered case). In the following of the present section, it will be briefly illustrated how the mentioned IKF technique allows to propagate Eq. (16) in its Gaussian approximated form, with a reasonable computational effort. To this aim, reconsider Eq. (16) and formerly observe how a maximum $a$ posteriori probability (MAP) estimate $\widehat{x}_{i / i}$ can be obtained by maximizing the right-hand side of Eq. (16) itself with respect to $x_{i}$; this is, in turn, equivalent to minimize the sum of the exponents in Eq. (16):

$$
\widehat{x}_{i / i}=\underset{x_{i}}{\operatorname{argmin}}\left[\left\|y_{i}-C\left({ }^{i-2} \theta_{i-1}\right) x_{i}\right\|_{S_{i}}^{2}+\left\|x_{i}-\widehat{x}_{i / i-1}\right\|_{P_{i / i-1}}^{2}\right] \text {. }
$$

Since the argument of the minimization in Eq. (17) is actually nontotally quadratic (still due to the nonlinear dependence of matrix $C\left({ }^{i-2} \theta_{i-1}\right)$ on the second component ${ }^{i-2} \theta_{i-1}$ of the whole state $x_{i}$ ), a recursive numerical gradienttype, Newton, or Newton-Raphson algorithm can be used to achieve the minimum point $\widehat{x}_{i / i}$ as in any nonquadratic minimization problem. Then, once the minimum point $\widehat{x}_{i / i}$ has been achieved, an approximate evaluation $P_{i / i}$ of the estimation error covariance can be obtained as the inverse Hessian matrix of the argument of Eq. (17), evaluated in $\widehat{x}_{i / i}$. In the case a gradient or Newton type procedure is employed, the analytical expression of the Hessian matrix can be a priori deduced from Eq. (17); its numerical value at $\widehat{x}_{i / i}$ has then to be extracted and inverted. Instead, in the case of a Newton-Raphson procedure, the numerical value $P_{i / i}(k)$ of the Hessian Inverse at the current iteration point $\widehat{x}_{i / i}(k)$ is provided by the structure of the procedure itself. Then, once $\widehat{x}_{i / i}$ and $P_{i / i}$ have been finally achieved, the Gaussian approximation 


$$
p\left(\frac{x_{i}}{I_{i}}\right) \approx N\left(x_{i}, \widehat{x}_{i / i}, P_{i / i}\right)
$$

is introduced to reach the next stage and so on. Within the literature, the usage of the Newton-Raphson procedure to minimize any nonquadratic cost resulting from a nonlinear (in its measurement equation only) system is generally termed as IKF, even if this terminology is also extended to the case of the employment of any other numerical minimization technique. Finally, it is worth recalling that, still in the case of a Newton-Raphson procedure but initialized at $\widehat{x}_{i / i-1}$ and also arrested at the first iteration, the resulting incomplete IKF procedure actually turns out to coincide with the previously seen EKF. This last fact clearly shows how IKF always result better, in terms of performances, than the EKF, thus indicating the IKF as the preferable one, provided that the available computing power on-board the vehicle is able to face it. Now, the present section should be concluded with pointing out some investigation directions that should be followed to best adapt the IKF procedure to the considered case. More precisely, by keeping into account the very particular structure attained by the output matrix $C\left({ }^{i-2} \theta_{i-1}\right)$, that is, from Eq. (6) and Eq. (8)

$$
C\left({ }^{i-2} \theta_{i-1}\right)=B \operatorname{diag}\left[C_{1}, C_{2}\left({ }^{i-2} \theta_{i-1}\right)\right]=B \operatorname{diag}\left\{\left[\begin{array}{ll}
0 & 1 \\
1 & 1
\end{array}\right],\left[\begin{array}{cc}
0 & 1 \\
1 & R\left({ }^{i-2} \theta_{i-1}\right)
\end{array}\right]\right\}
$$

with $R\left({ }^{i-2} \theta_{i-1}\right)$ as in Eq. (2), a first investigation direction could be that of trying to exploit such a peculiarity to achieve a best structure for the associate Newton-Raphson algorithm working on the whole state $x_{i}$.

As a second investigation direction, one could instead consider the minimization problem embedded in Eq. (17), once rewritten as:

$$
\min _{i-2}\left\{\min _{x_{i} /{ }^{i-2} \theta_{i-1}}\left[\left\|y_{i}-C\left({ }^{i-2} \theta_{i-1}\right) x_{i}\right\|_{S_{i}}^{2}+\left\|x_{i}-\hat{x}_{i / i-1}\right\|_{P_{i / i-1}}^{2}\right]\right\} .
$$

Noting that for given ${ }^{i-2} \theta_{i-1}$, the inner minimization turns out to be quadratic, an analytical linear expression for the resulting conditioned minimum point $\widehat{x}_{i / i}\left({ }^{i-2} \theta_{i-1}\right)$ must necessarily be obtained, also exploiting the particular structure of $C\left({ }^{i-2} \theta_{i-1}\right)$ as

$$
\widehat{x}_{i / i}\left({ }^{i-2} \theta_{i-1}\right)=L\left[R\left({ }^{i-2} \theta_{i-1}\right), y_{i}, \hat{x}_{i / i-1}\right]
$$

where the symbol $L$ just stands for linear and which is actually valid in correspondence of any stage. The linear form in Eq. (20), once substituted into (19), must necessarily lead to an analytical expression for the resulting value of its inner conditioned optimal cost, say $J\left({ }^{i-2} \theta_{i-1}, y_{i}, \hat{x}_{i / i-1}\right)$, within Eq. (19) itself; the residual minimization is thus reduced to $\min _{x_{i} /{ }^{i-2} \theta_{i-1}} J\left({ }^{i-2} \theta_{i-1}, y_{i}, \hat{x}_{i / i-1}\right)$ again valid in correspondence of any stage and whose numerical minimization 
now results very much simplified since it is performed along a one-dimensional set. Note, however, that, regarding the estimated error covariance, this should be instead evaluated at $\widehat{x}_{i / i}$ via the Hessian matrix analytical expression a priori deduced from the argument of Eq. (17) and exploiting again the particular structure of Eq. (18). Finally, as a third investigation alternative, it would be also taken into account the possibility (however, very unlikely) that the analytical expression of $J\left({ }^{i-2} \theta_{i-1}, y_{i}, \hat{x}_{i / i-1}\right)$ might also result in an "easy-to-bezeroed" partial derivative with respect to its first argument ${ }^{i-2} \theta_{i-1}$. This last fact, whenever true for the considered case, would actually reduce the associated quasi-optimal IKF state estimation procedure to the simple and very fast application of some invariant analytical formulas at each stage, with an enormous advantage in terms of required computational power.

\subsection{Remarks}

Both the IKF-type State Estimators (STE) and its reduced EKF version are the recursive filters that produce the current stage state estimation updating their prediction, in turn obtained from the previous estimations. Hence, since the error occurred at any stage will not change in the future and since the absolute vehicle position and orientation has to be evaluated still via Eq. (1), it is clear that linearly increasing errors have to be expected for increasing number of stages (with the only difference that such a growth is now mitigated by the reduced variances of the relative errors, due to the presence of state estimator). Obviously enough, things might be different in case the incoming acquisitions for increasing stages could be used to produce not only the current state estimate, but also to better, at the same time, all the past state estimations. As a matter of fact, this objective can be achieved via the use of the so-called Sequence Estimation (SQE) techniques, as discussed in section 4.

\section{SEQUENCE ESTIMATION}

In order to start facing the argument, let first introduce some notations to denote the sequences of the true relative orientations and positions, realized up to the $i$ th stage

$$
\begin{aligned}
& \theta^{1, i} \doteq\left\{{ }^{k-1} \theta_{k} ; k=1,2, \ldots, i\right\} \\
& r^{1, i} \doteq\left\{{ }^{k-1} r_{k} ; k=1,2, \ldots, i\right\}
\end{aligned}
$$

together with the aggregation of the two

$$
X^{1, i} \doteq\left\{\theta^{1, i} ; r^{1, i}\right\}
$$


and also the following expressions to indicate the associated sequences of the collected measurements

$$
\begin{aligned}
z^{1, i} & \doteq\left\{\left({ }^{k-1} \hat{\theta}_{k},{ }^{k-2} \hat{\theta}_{k}\right) ; k=1,2, \ldots, i\right\} \\
w^{1, i} & \doteq\left\{\left({ }^{k-1} \hat{r}_{k},{ }^{k-2} \hat{r}_{k}\right) ; k=1,2, \ldots, i\right\}
\end{aligned}
$$

aggregated as well into

$$
Z^{1, i} \doteq\left\{z^{1, i} ; w^{1, i}\right\}
$$

Once the $i$ th stage has been achieved, the problem to be solved consists in finding the overall sequence estimate:

$$
\widehat{X}^{1, i}=\left\{\widehat{\theta}^{1, i} ; \widehat{r}^{1, i}\right\}=\underset{X^{1, i}}{\operatorname{argmax}} p\left(\frac{X^{1, i}}{Z^{1, i}}\right) .
$$

As it can be realized, provided that the problem is solvable (even in an approximate or suboptimal form), its solution will renew, at each stage $i$, a batch of interpolated estimations of the entire sequence $X^{1, i}$ realized before it, without relying on the estimate of the previous one $X^{1, i-1}$. Due to this, one may argue about the drift errors, generally characterizing IKF and EKF techniques: they should, in this case, result strongly reduced. Still assuming the problem solvability, an obvious drawback is, however, represented by the apparent increasing dimensionality with the increasing number of stages. The dimensionality growth is actually linear and, so, it is quite acceptable within a reasonably high maximum number of stages, after which the obtained sequence estimate should be "freezed" and the entire procedure restarted along the successive maximum horizon of stages. At this point, in order to manage the problem, however, the cascade of the following subproblems should be considered:

$$
\overbrace{\theta}^{1, i} \doteq \underset{\theta^{1, i}}{\operatorname{argmax}} p\left(\frac{\theta^{1, i}}{z^{1, i}}\right) \rightarrow \widehat{r}^{1, i} \doteq \underset{r^{1, i}}{\operatorname{argmax}} p\left(\frac{r^{1, i}}{w^{1, i}}, \widehat{\theta}^{1, i}\right)
$$

where the orientation sequence is estimated on the basis of its own measurements only, while the position sequence is evaluated in cascade, on the basis of its measurements and taking $\overbrace{}^{1, i}$ as a given parameter set. The need to structure the problem in the above suboptimal way, with respect to the original one, actually arises from the necessity to maintain a manageable implementative form that otherwise cannot be guaranteed, whenever the problem is dealt with as a whole, like in Eq. (21). Hence, considering the first subproblem, formerly observe how, via a standard application of the Bayes rule, for the involved p.d.f., one actually has 


$$
\begin{aligned}
p\left(\frac{\theta^{1, i}}{z^{1, i}}\right)= & \frac{1}{M_{1, i}} p\left(\frac{z^{1, i}}{\theta^{1, i}}\right)=\prod_{k=2}^{i} \exp \left[-\frac{\left|{ }^{k-2} \theta_{k-1}-{ }^{k-2} \hat{\theta}_{k-1}\right|^{2}}{k-2} \Theta_{k-1}\right. \\
& \left.-\frac{\left|\left({ }^{k-2} \theta_{k-1}+{ }^{k-1} \hat{\theta}_{k}\right)-{ }^{k-2} \hat{\theta}_{k}\right|^{2}}{{ }^{k-2} \Theta_{k}}+\frac{\left|k-1 \theta_{k}-{ }^{k-1} \hat{\theta}_{k}\right|^{2}}{k-1 \Theta_{k}}\right]
\end{aligned}
$$

where the first equality simply states that the posterior p.d.f. $p\left(\theta^{1, i} / z^{1, i}\right)$ substantially coincides with the maximum likelihood one $p\left(z^{1, i} / \theta^{1, i}\right)$ since the prior $p\left(\theta^{1, i}\right)$ is actually uniform; on the other hand, the second equality follows directly from the assumed independence and Gaussianity of the added measurement noises. Then, note how maximizing Eq. (23) is equivalent to the following minimization problem:

$$
\begin{aligned}
& \widehat{\theta}^{1, i} \doteq \underset{\theta^{1, i}}{\operatorname{argmin}} \sum_{k=2}^{i}\left[\frac{\left|k-2 \theta_{k-1}-{ }^{k-2} \hat{\theta}_{k-1}\right|^{2}}{k-2} \Theta_{k-1}\right. \\
& \left.+\frac{\left|\left({ }^{k-2} \theta_{k-1}+{ }^{k-1} \hat{\theta}_{k}\right)-{ }^{k-2} \hat{\theta}_{k}\right|^{2}}{{ }^{k-2} \Theta_{k}}+\frac{\left|{ }^{k-1} \theta_{k}-{ }^{k-1} \hat{\theta}_{k}\right|^{2}}{{ }^{k-1} \Theta_{k}}\right] \\
& \doteq \underset{\theta^{1, i}}{\operatorname{argmin}} J^{1, i}\left(\theta^{1, i}\right) .
\end{aligned}
$$

Moreover, since by isolating the first stage Eq. (24) can be equivalently rewritten as

$$
\begin{array}{r}
\min _{\theta^{2, i}}\left\{\min _{{ }^{0} \theta_{1}}\left[\frac{\left|{ }^{0} \theta_{1}-{ }^{0} \hat{\theta}_{1}\right|^{2}}{{ }^{0} \Theta_{1}}+\frac{\left|\left({ }^{0} \theta_{1}+{ }^{1} \theta_{2}\right)-{ }^{0} \hat{\theta}_{2}\right|^{2}}{{ }^{0} \Theta_{2}}\right]_{/{ }^{1} \theta_{2}}\right. \\
\left.+\frac{\left|{ }^{1} \theta_{2}-{ }^{1} \hat{\theta}_{2}\right|^{2}}{{ }^{1} \Theta_{2}}+J^{2, i}\left(\theta^{2, i}\right)\right\}
\end{array}
$$

(being $J^{2, i}\left(\theta^{2, i}\right)$ the same of Eq. (24) even if now starting from $k=3$ ), it then follows that within Eq. (25), one can actually proceed as hereafter indicated.

1. Minimize the first part for given ${ }^{1} \theta_{2}$. Since the expression to be minimized is quadratic, the result will be linear in ${ }^{1} \theta_{2}$; the following linear parameterization is thus obtained:

$$
{ }^{0} \widehat{\theta}_{1}=L_{1}\left({ }^{1} \theta_{2}\right)
$$


2. Substitute the result of Eq. (26) into (25) - the result will necessary be a quadratic function of ${ }^{1} \theta_{2}$ - and define:

$$
Q_{1}\left({ }^{1} \theta_{2}\right) \doteq \min _{{ }^{0} \theta_{1}}\left[\frac{\left|{ }^{0} \theta_{1}-{ }^{0} \hat{\theta}_{1}\right|^{2}}{{ }^{0} \Theta_{1}}+\frac{\left|\left({ }^{0} \theta_{1}+{ }^{1} \theta_{2}\right)-{ }^{0} \hat{\theta}_{2}\right|^{2}}{{ }^{0} \Theta_{2}}\right]_{/{ }^{1} \theta_{2}}
$$

3. Extract from $J^{2, i}\left(\theta^{2, i}\right)$ its first three terms. Group the first two with $Q_{1}\left({ }^{1} \theta_{2}\right)$ - naming it $\bar{Q}_{2}\left({ }^{1} \theta_{2},{ }^{2} \theta_{3}\right)$ - and obtain

$$
\min _{\theta^{2, i}}\left\{\bar{Q}_{2}\left({ }^{1} \theta_{2},{ }^{2} \theta_{3}\right)+\frac{\left|{ }^{2} \theta_{3}-{ }^{2} \hat{\theta}_{3}\right|^{2}}{{ }^{2} \Theta_{3}}+J^{3, i}\left(\theta^{3, i}\right)\right\}
$$

where $J^{3, i}\left(\theta^{3, i}\right)$ is obviously the same as in $(25)$ even if now starting from $k=4$ and where by construction

$$
\bar{Q}_{2}\left({ }^{1} \theta_{2},{ }^{2} \theta_{3}\right)=Q_{1}\left({ }^{1} \theta_{2}\right)+\frac{\left|{ }^{1} \theta_{2}-{ }^{1} \hat{\theta}_{2}\right|^{2}}{{ }^{1} \Theta_{2}}+\frac{\left|\left({ }^{1} \theta_{2}+{ }^{2} \theta_{3}\right)-{ }^{1} \hat{\theta}_{3}\right|^{2}}{{ }^{1} \Theta_{3}} .
$$

4. Finally, rewrite (27) similarly to (25) as

$$
\min _{\theta^{3, i}}\left\{\min _{{ }^{1} \theta_{2}} \bar{Q}_{2}\left({ }^{1} \theta_{2},{ }^{2} \theta_{3}\right)_{/ 1} \theta_{2}+\frac{\left|{ }^{2} \theta_{3}-{ }^{2} \hat{\theta}_{3}\right|^{2}}{{ }^{2} \Theta_{3}}+J^{3, i}\left(\theta^{3, i}\right)\right\}
$$

and return to the first point, repeating all the steps with respect to (28) and so on for all successive stages.

Note that the linear parameterization ${ }^{k-1} \widehat{\theta}_{k}=L_{k}\left({ }^{k} \theta_{k+1}\right)$ at the current stage $k$ represents the constraint for the previous stage, according to the back substitution scheme depicted in Fig. 5. When the $i$ th stage is finally reached assuming that no more measurements are acquired (or equivalently assuming that the procedure has to be stopped at such a stage), the last minimization problem - considering that in correspondence of the last stage $J^{i, i}\left(\theta^{i, i}\right) \doteq 0-$ can be written as $\min _{\theta^{i-1, i}}\left\{\bar{Q}_{i-1}\left({ }^{i-2} \theta_{i-1},{ }^{i-1} \theta_{i}\right)+\left|{ }^{i-1} \theta_{i}-{ }^{i-1} \hat{\theta}_{i}\right|^{2} /{ }^{i-1} \Theta_{i}\right\}$. Such a minimization can be here performed directly with respect to both ${ }^{i-2} \theta_{i-1}$ and ${ }^{i-1} \theta_{i}$, thus obtaining their estimations ${ }^{i-2} \widehat{\theta}_{i-1}$ and ${ }^{i-1} \widehat{\theta}_{i}$. Hence, such numerical values can be used to realize the above back substitution scheme; this procedure actually corresponds to the well known Forward Dynamic Programming (FDP) 


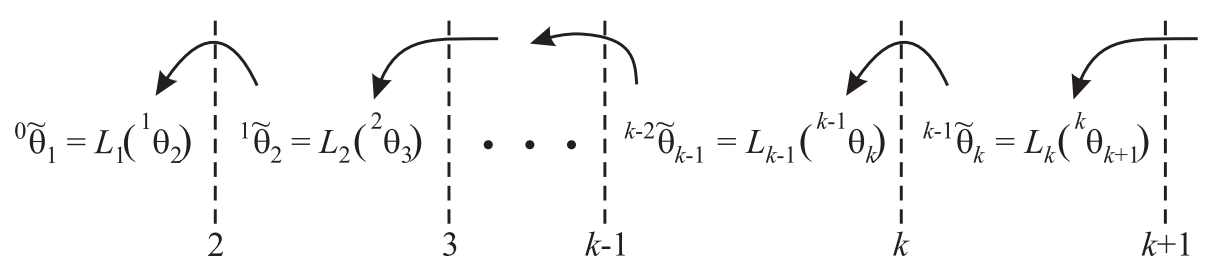

Figure 5 Back substitution scheme

technique, here applied to sequence estimation problems. This method requires to wait until the last $i$ th stage before the estimate of the orientation sequence realized till such stage is provided. Nevertheless, it could also be implemented in such a way that, while the forward phase proceeds, the backward phase is, however, executed in correspondence to any new incoming stage. In this way, the procedure would totally renew the interpolated sequence in correspondence to each new arrived stage till the last one. Note how the sole backward phase is the one working with a computational effort increasing with the number of stages; thus, a limit to the maximum number of processable stages should be substantially established only on this basis. Once the farthest allowed stage is reached, the procedure can be restarted by trivially considering such farthest stage as the new initial one. In this way, drifting errors occurring within a cascade of long stage batches would result much more contained than in the case of state filtering techniques. Considering now the subproblem of estimating the position sequence, just observe that (again via the standard Bayes rule)

$$
\begin{aligned}
& p\left(\frac{r^{1, i}}{w^{1, i}}, \widehat{\theta}^{1, i}\right)=\frac{1}{H_{1, i}} p\left(\frac{w^{1, i}}{r^{1, i}}, \widehat{\theta}^{1, i}\right) p\left(r^{1, i}\right)=p\left(\frac{w^{1, i}}{r^{1, i}}, \widehat{\theta}^{1, i}\right) \\
&= \prod_{k=2}^{i} \exp \left[-\left\|^{k-2} r_{k-1}-{ }^{k-2} \hat{r}_{k-1}\right\|_{k-2}^{2} \Sigma_{k-1}^{-1}\right. \\
&-\left\|\left[{ }^{k-2} r_{k-1}+R\left({ }^{k-2} \widehat{\theta}_{k-1}\right){ }^{k-1} r_{k}\right]-{ }^{k-2} \hat{r}_{k}\right\|_{k-2}^{2} \Sigma_{k}^{-1} \\
&\left.-\left\|{ }^{k-1} r_{k}-{ }^{k-1} \hat{r}_{k}\right\|_{k-1}^{2} \Sigma_{k}^{-1}\right]
\end{aligned}
$$

where, as assumed within the (suboptimal) problem statement in Eq. $(22), \widehat{\theta^{1, i}}$ has to be taken as a given set of parameters without any probabilistic characterization. Then, under this assumption, the first line of Eq. (29) again states that the posterior p.d.f. substantially coincides with the maximum likelihood one, since the prior p.d.f. is actually uniform. Moreover, Eq. (29) holds thanks to the assumed independence and Gaussianity of the added measurement noises. Then, just as before, maximizing Eq. (29) is equivalent to the following minimum problem: 


$$
\begin{aligned}
\widehat{r}^{1, i} \underset{r^{1, i}}{\operatorname{argmin}} \sum_{k=2}^{i}\left[\left\|{ }^{k-2} r_{k-1}-{ }^{k-2} \hat{r}_{k-1}\right\|_{k-2}^{2} \Sigma_{k-1}^{-1}\right. \\
+\left\|\left[{ }^{k-2} r_{k-1}+R\left({ }^{k-2} \widehat{\theta}_{k-1}\right){ }^{k-1} r_{k}\right]-{ }^{k-2} \hat{r}_{k}\right\|_{k-2 \Sigma_{k}^{-1}}^{2} \\
\left.+\left\|^{k-1} r_{k}-{ }^{k-1} \hat{r}_{k}\right\|_{k-1}^{2} \Sigma_{k}^{-1}\right] \underset{r^{1, i}}{\operatorname{argmin}} H^{1, i}\left(r^{1, i}\right) .
\end{aligned}
$$

Since for given $\overbrace{}^{1, i}$ the functional to be minimized in Eq. (30) actually exhibits the same structure of its analogous in Eq. (24), all considerations provided for the previous problem in Eq. (24) can be again applied to Eq. (30), thus leading, also for the positions, to a strictly similar FDP-based sequence estimation algorithm. This actually follows from the assumption of considering the angular sequence estimates $\overbrace{}^{1, i}$ as a given parameter set: suboptimality is accepted, since otherwise a joint estimation (very difficult for the nonlinearity) of both sequences $\theta^{1, i}$ and $r^{\nu, i}$ would be required. Moreover, a drawback of such an implementation is unfortunately exhibited by the sequence position estimation and consists in the need of waiting till the $i$ th stage for acquiring $\widehat{\overbrace{}}^{1, i}$ and executing the position forward and backward phases. Hence, in order to renew $\overbrace{}^{1, i-1}$ and $\widehat{r}^{1, i-1}$ into $\widehat{\sigma}^{1, i}$ and $\widehat{r}^{1, i}$, the following operations must be achieved at each stage.

1. Upgrade the forward phase of the orientation sequence estimator.

2. Perform its entire backward phase, thus obtaining $\overbrace{}^{1, i}$.

3. Perform the entire forward phase of the position sequence estimator, given $\overbrace{}^{1, i}$.

4. Perform its entire backward phase, thus obtaining $\widehat{r}^{1, i}$.

This actions must be performed until a maximum allowed distance $j$-stage is achieved, after which a new sequence is restarted. In the following section, it will be shown how the integration of a state estimator with a sequence one might further improve the overall estimation performances, while also allowing an efficient management of the available computing power.

\section{EXPLOITING MULTIPLE SENSORS}

Generally speaking, multisensor integration means suitable exploitation of different sensors providing measures of the same set of variables, in order to obtain 

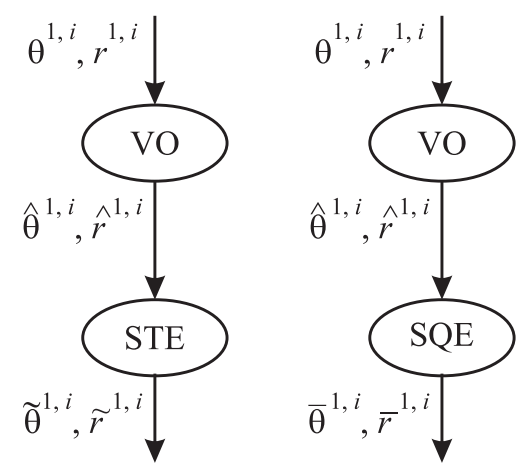

Figure 6 Alternative basic integration schemes

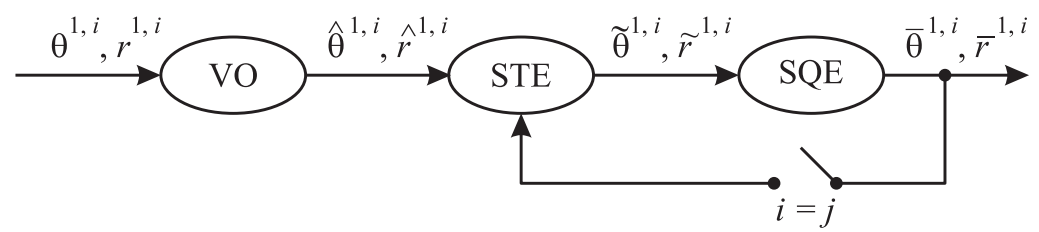

Figure 7 A preliminary tentative integration scheme

an augmented sensor characterized by better performances. In this sense, the VO technique can be seen as a component sensor that, based on its own internal procedures, at each stage $i$ adds the set of measurements ${ }^{i-2} \hat{\theta}_{i-1},{ }^{i-1} \hat{\theta}_{i}$, ${ }^{i-2} \hat{r}_{i-1}$, and ${ }^{i-1} \hat{r}_{i}$ (plus the auxiliary ones ${ }^{i-2} \hat{\theta}_{i}$ and ${ }^{i-2} \hat{r}_{i}$ ) of the associated variables to the collection $\hat{\theta}^{1, i-1}$ and $\hat{r}^{1, i-1}$ of the identical measurements (here also including the auxiliary ones) acquired till the previous stage. Then, accordingly with the previous section, the VO sensor can be integrated with a state estimator (EKF or IKF) or, alternatively, with a sequence estimator, in order to obtain better performances in terms of the variances of the produced estimates (they will be now called $\tilde{\theta}^{1, i-1}$ and $\tilde{r}^{1, i-1}$ for the state estimators and $\bar{\theta}^{1, i-1}$ and $\bar{r}^{1, i-1}$ for the sequence ones). Besides the two separate schemes shown in Fig. 6, it could be interesting try to combine these two alternatives together, obtaining a tentative scheme for the integration of all the three components, as in Fig. 7 (neglect for a while the indicated feedback loop). The rationale for such a scheme follows immediately from the intuitive idea that, being the SQE module fed with measurements characterized by smaller variances than those provided by the VO module alone, it should, in turn, provide SQE with further smaller variances. Moreover, the indicated feedback loop could be used when the maximum allowed distance is achieved, in order to reinitial- 
ize the STE module with $\bar{\theta}^{1, j}$ and $\bar{r}^{1, j}$, that is with the hereafter indicated terms

$$
\left\{\begin{array} { l } 
{ ( { } ^ { j - 2 } \overline { \theta } _ { j - 1 } , { } ^ { j - 1 } \overline { \theta } _ { j } ) } \\
{ ( { } ^ { j - 2 } \overline { r } _ { j - 1 } , { } ^ { j - 1 } \overline { r } _ { j } ) }
\end{array} \rightarrow \left\{\begin{array}{l}
{ }^{j-2} \bar{\theta}_{j}={ }^{j-2} \bar{\theta}_{j-1}+{ }^{j-1} \bar{\theta}_{j} ; \\
{ }^{j-2} \bar{r}_{j}={ }^{j-2} \bar{r}_{j-1}+R\left({ }^{j-2} \bar{\theta}_{j-1}\right)^{j-1} \bar{r}_{j}
\end{array}\right.\right.
$$

in such a way to allow the STE module to restart from stage $j$ with better initial conditions and then to repeat the entire process for the next batch of stages of maximum length $j$. Within the above tentative scheme, the idea is to use the STE module to know (even if less accurately) the vehicle position with respect to the last estimation produced by the SQE.

Unfortunately enough, however, this integrated scheme produces neither any bettering nor any worsening with respect to the STE estimation alone. In fact, since the SQE is fed with the STE-provided measurement sequence $\bar{\theta}^{1, j}$ and $\bar{r}^{1, j}$, where, in correspondence with each sequential couple of its component it should be also evaluated (similarly to Eq. (31) but in correspondence to each intermediate stage $i$ till the $j$ th one)

$$
\begin{aligned}
& \left\{\begin{array} { l } 
{ ( { } ^ { i - 2 } \widehat { \theta } _ { i - 1 } , { } ^ { i - 1 } \widehat { \theta } _ { i } ) } \\
{ ( { } ^ { i - 2 } \widehat { r } _ { i - 1 } , { } ^ { i - 1 } \widehat { r } _ { i } ) }
\end{array} \rightarrow \left\{\begin{array}{l}
{ }^{i-2} \widehat{\theta}_{i}={ }^{i-2} \widehat{\theta}_{i-1}+{ }^{i-1} \widehat{\theta}_{i} \\
i-2 \widehat{r}_{i}={ }^{i-2} \widehat{r}_{i-1}+R\left(i-2 \widehat{\theta}_{i-1}\right) i-1 \\
\widehat{r}_{i}
\end{array}\right.\right. \\
& i=1,2, \ldots, j,
\end{aligned}
$$

it is easy to see that the corresponding forms of Eqs. (25) and (30), to be minimized inside the SQE, are both zeroed (thus absolutely minimized) just by the same STE-provided sequence: this makes the first suggested scheme totally useless. On the other hand, this result is totally coherent with the fact that, once the information has been processed by the STE, the estimation cannot at all be improved without adding more information (i. e., without acquiring more data). Hence, STE and SQE can be integrated only if they run in parallel, as indicated in Fig. 8.

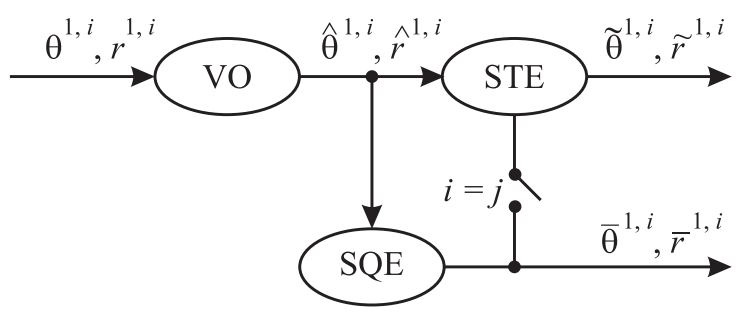

Figure 8 The correct integration scheme 
Now, the correct integration scheme between STE end SQE has been established; so, it is possible to proceed further on by also adding new sensors, like, for instance, IMU and/or classical WO sensor systems (generally present on board most vehicles), which could improve both the STE and SQE modules.

\section{State Estimator Improvement}

Firstly, consider the case of an IMU system located on board the vehicle; such a sensor provides (substantially in a continuous-time manner) measurements about the angular velocity vector $\omega$ and the linear acceleration vector $\dot{v}$, both projected on the vehicle frame. Since it is now assumed the planar motion only (more general cases will be subjected to further studies), the angular velocity vector will always be about the vertical axis and so its projection on both the vehicle and the absolute frame is the same (it will be simply indicated as $\omega$ ). On the other hand, the linear acceleration vector, still due to the planar motion assumption, will always lie on the horizontal plane (there will be denoted its two-dimensional projection on the horizontal plane of the vehicle frame simply as vector $a$ ). Thus, the continuous time (typically noisy) measurements provided by an IMU system are typically of the form

$$
\tilde{\omega}(t)=\omega(t)+e_{1}(t) ; \quad \tilde{a}(t)=a(t)+e_{2}(t)
$$

with $e_{1}(t), e_{2}(t)$ assumed continuous-time zero mean white stationary Gaussian noises (for simplicity, also uncorrelated between them) with covariance functions $E_{1} \delta(\tau)$ and $E_{2} \delta(\tau)$, respectively, where $\delta(\tau)$ is the Dirac unit impulse function. In particular, note how, from a kinematic point of view, the IMU actually provides first-order information for the orientation (the angular velocity measurements $\tilde{\omega}(t))$ and second-order one for the positions (the linear acceleration measurements $\tilde{a}(t))$. Considering now a generic stage $i$ and the associated time instant $t_{i}$ in correspondence of which the last measurement acquisition by the VO has been performed, the following integral process, starting at the instant $t_{i-1}$

$$
{ }^{i-1} \tilde{\theta}(t) \doteq \int_{t_{i-1}}^{t} \tilde{\omega} d t={ }^{i-1} \theta(t)+{ }^{i-1} \mu(t)
$$

with

$$
\begin{gathered}
{ }^{i-1} \theta(t) \doteq \int_{t_{i-1}}^{t} \omega(t) d t \\
{ }^{i-1} \mu(t) \doteq \int_{t_{i-1}}^{t} e_{1}(t) d t ; \quad{ }^{i-1} M(t)=E_{1}\left(t-t_{i-1}\right)
\end{gathered}
$$


provides a continuous-time measure of the vehicle orientation with respect to frame $\langle i-1\rangle$, while moving between time instants $t_{i-1}$ and $t_{i}$. Moreover, such an estimation becomes an additional measurement ${ }^{i-1} \tilde{\theta}_{i}$ of the angle ${ }^{i-1} \theta_{i}$ at time $t=t_{i}$, namely:

$$
{ }^{i-1} \tilde{\theta}_{i}={ }^{i-1} \theta_{i}+{ }^{i-1} \mu_{i} ; \quad{ }^{i-1} M_{i}=E_{1}\left(t_{i}-t_{i-1}\right) \doteq E_{1} T_{i} .
$$

In Eq. (32), it can be noted how the superimposed equivalent noise ${ }^{i-1} \mu(t)$ obviously results into a zero mean Wiener process (i. e., a so-called random walk with independent increments), exhibiting the linearly time increasing covariance matrix indicated in the second term of Eq. (32) and, in turn, becoming the one in Eq. (33) at time $t_{i}$. Further, note how the equivalent noise sequence ${ }^{i-1} \mu_{i}$ in Eq. (33) results into a zero mean white Gaussian one - with covariance as in Eq. (33) - since it is composed by a sequence of disjoint integrals of the continuous time zero mean white Gaussian noise $e_{1}(t)$ (as a matter of fact, it would have been sufficient to have the noise $e_{1}(t)$ uncorrelated for time delays greater than the integration interval $T_{i}$ ). Similarly, for the continuous time measurement $\tilde{a}$, recalling relationship of Eq. (3), observe that, kinematically,

$$
\begin{aligned}
{ }^{i-2} r_{i}={ }^{i-2} r_{i-1}+R\left({ }^{i-2} \theta_{i-1}\right) & { }^{i-1} r_{i} \\
= & { }^{i-2} r_{i-1}+T_{i}{ }^{i-2} v_{i-1}+R\left({ }^{i-2} \theta_{i-1}\right){ }^{i-1} h_{i}^{2}
\end{aligned}
$$

where ${ }^{i-2} v_{i-1}$ is the vehicle velocity at time $t_{i-1}$ projected on frame $\langle i-2\rangle$; moreover, the following equation shows the double integral of the true vehicle acceleration projected on frame $\langle i-1\rangle$ during the time interval $t_{i-1}, t_{i}$, just corresponding to the contribution to the position with respect to $\langle i-1\rangle$ provided by the acceleration itself:

$$
{ }^{i-1} h_{i}^{2} \doteq \int_{t_{i-1}}^{t_{i}} d \tau \int_{t_{i-1}}^{\tau} R\left[^{i-1} \theta(\tau)\right] a(\tau) d \tau .
$$

Furthermore, note that for the velocity ${ }^{i-2} v_{i-1}$, kinematically, one has

$$
{ }^{i-2} v_{i-1}={ }^{i-2} v_{i-2}+{ }^{i-2} h_{i-1}^{1}
$$

with

$$
{ }^{i-2} h_{i-1}^{1} \doteq \int_{t_{i-2}}^{t_{i-1}} R\left[{ }^{i-2} \theta(\tau)\right] a(\tau) d \tau
$$

which is the integral of the true vehicle acceleration projected on frame $\langle i-2\rangle$, during the time interval $t_{i-2}, t_{i-1}$, corresponding to the contribution to the velocity provided by the acceleration itself. 
Since for ${ }^{i-2} r_{i-1}$ kinematically one has

$$
{ }^{i-2} r_{i-1}=T_{i-1}{ }^{i-2} v_{i-2}+{ }^{i-2} h_{i-1}^{2},
$$

then, by deducing ${ }^{i-2} v_{i-2}$ from Eq. (36), substituting it into Eq. (35), and finally substituting the result into the second of Eq. (34), with very simple algebra, one gets the linear form:

$$
{ }^{i-2} r_{i}=c_{i-1}^{0}{ }^{i-2} r_{i-1}+c_{i-1}^{1}{ }^{i-2} h_{i-1}^{1}+c_{i-1}^{2}{ }^{i-2} h_{i-1}^{2}+R\left({ }^{i-2} \theta_{i-1}\right){ }^{i-1} h_{i}^{2} .
$$

At this point, still keeping into account the first of Eqs. (34), one can operate the following definitions:

$$
\begin{aligned}
y_{i} \doteq c_{i-1}^{1}{ }^{i-2} h_{i-1}^{1} & +c_{i-1}^{2}{ }^{i-2} h_{i-1}^{2}+R \\
=\left(1-c_{i-1}^{0}\right)^{i-2} r_{i-1}+R\left({ }^{i-2} \theta_{i-1}\right){ }^{i-1} \theta_{i-1} h_{i}^{2} & { }^{i-1} r_{i} \\
& \doteq \alpha_{i-1}{ }^{i-2} r_{i-1}+R\left({ }^{i-2} \theta_{i-1}\right){ }^{i-1} r_{i}
\end{aligned}
$$

As one can see, the left-hand side, $y_{i}$, of Eq. (37) actually constitutes a global quantity which can be noisily provided by the IMU sensor system. Consequently, provided that one can characterize the so-obtained measurement $\tilde{y}_{i}$ in terms of added noise to the true value $y_{i}$ itself (that is, in the form $\tilde{y}_{i}=y_{i}+\sigma_{i}$ with $\sigma_{i}$ possibly white with known covariance), the following relationship can be written:

$$
\tilde{y}_{i}=\alpha_{i-1}{ }^{i-2} r_{i-1}+R\left({ }^{i-2} \theta_{i-1}\right){ }^{i-1} r_{i}+\sigma_{i} .
$$

It then becomes clear how Eq. (38), together with Eq. (33), once repeated for both stages $i$ and $i-1$, results in three additional output relationships enriching the set of available measurements. Nevertheless, to this regard, observe how the terms ${ }^{i-1} h_{i}^{2},{ }^{i-2} h_{i-1}^{1}$, and ${ }^{i-2} h_{i-1}^{2}$ composing $y_{i}$ in Eq. (37), have been represented considering $a(t)$ and $\omega(t)$ as completely unrelated, while this is instead not the case for nonholonomic vehicles (as planetary rovers most probably are). As a matter of fact, $n$ on-holonomic constraints might probably introduce simplifications; this fact should be better investigated. Finally, it should be also remarked that the analytical steps which lead to Eqs. (37) and (38) were actually necessary in order to eliminate from the considered kinematic equations all the linear velocity terms (since neither directly measured nor part of the adopted state space model). The integrated overall scheme improving the STE module with the inclusion of the IMU system, obviously results into the one reported in Fig. 9. Note, however, how the scheme also anticipates the presence of a block relative to the WO that can be integrated into the overall system as well. A WO system can directly provide, in a substantially continuous-time manner, the measurements $\breve{\omega}, \breve{v}$ of the vehicle angular velocity $\omega$ and its velocity vector $v$ projected on the vehicle fixed frame. These WO estimates are realtime obtained from the measured rotation velocities of the vehicle wheels and 


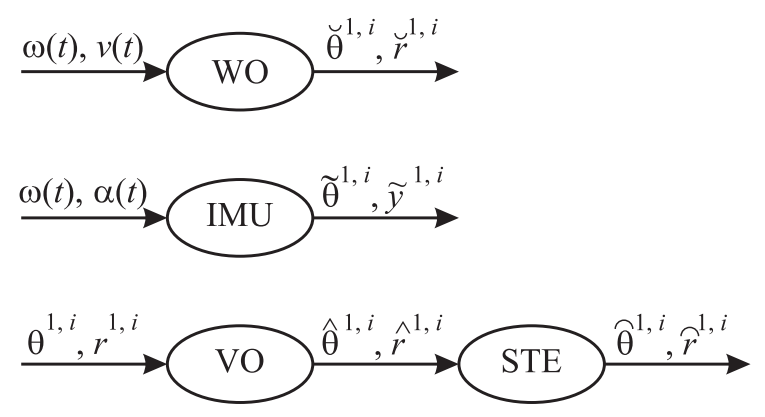

Figure 9 Improving the State Estimator

are generally handled within a specific (internal to the WO system itself) least square algorithm or even a specific state estimator. The WO module continuous time measurements can be expressed as

$$
\begin{aligned}
& \breve{\omega}(t)=\omega(t)+n_{1}(t) ; \\
& \breve{v}(t)=v(t)+n_{2}(t)
\end{aligned}
$$

where $n_{1}(t)$ and $n_{2}(t)$ are continuous-time zero mean white stationary Gaussian noises with covariance functions $N_{1} \delta(\tau)$ and $N_{2} \delta(\tau)$, respectively. In particular, note that, since the WO module actually provides first-order measurements for both orientation and position, for the angular velocity measurement in Eq. (39), similarly to Eq. (33), one can write:

$$
{ }^{i-1} \breve{\vartheta}_{i}={ }^{i-1} \theta_{i}+{ }^{i-1} \rho_{i} ; \quad{ }^{i-1} \theta_{i} \doteq \int_{t_{i-1}}^{t_{i}} \omega(t) d t
$$

where the resulting equivalent additional sequence noise (zero mean white Gaussian) can be expressed as

$$
{ }^{i-1} \rho_{i} \doteq \int_{t_{i-1}}^{t_{i}} n_{1}(t) d t ; \quad{ }^{i-1} Z_{i}=N_{1}\left(t_{i}-t_{i-1}\right)=N_{1} T_{i} .
$$

Moreover, from the first-order kinematics, one has:

$$
{ }^{i-1} r_{i}=\int_{t_{i-1}}^{t_{i}} R\left[{ }^{i-1} \theta(t)\right] v(t) d t
$$

simply corresponding to the integral of the velocity projected on $\langle i-1\rangle$; since such integral results into a (noisy) quantity that can be measured by the WO 
system, provided that its resulting measure ${ }^{i-1} \breve{r}_{i}$ can be characterized in terms of added noise, that is, as

$$
{ }^{i-1} \breve{r}_{i}={ }^{i-1} r_{i}+{ }^{i-1} \lambda_{i},
$$

it then follows that Eqs. (40) and (41), once considered for both stages $i$ and $i-1$, actually result into further six additional measurements to be used to improve the estimation.

In this last part of the work, it is described how to integrate multiple sensors in the proposed scheme exploiting state estimation only; note, however, that the same procedure can be adopted and the integration paradigm can be easily extended to the sequence estimation algorithm as well (details are omitted for the sake of brevity).

\section{CONCLUDING REMARKS AND COMMENTS}

Within this paper, a technique to improve rover localization has been suggested; this method employs an VO algorithm that has been previously developed at GRAAL Lab. Such a technique can result more efficient, above all for planetary scenarios, since, in general, it is less affected, with respect to other on-board sensors, by terrain roughness and slopes that can cause slippage. Visual odometry, as explained, can be improved through the action of state or sequence estimators; furthermore, since a rover is generally endowed also with other sensors, such as classical WO or IMU, a multisensory integration scheme has been proposed.

Anyway, some open issues have been pointed out: first of all, this idea is still under consideration and many simulations and experimental tests have to be carried out. Then, as already suggested, the method is to be extended to more wide motion cases, that is, foreseeing not only planar rover movements. Moreover, the method probably might be simplified, mostly taking into account the mentioned nonholonomic constraints for the rover. Finally, it should be noted how the devised multisensor integration scheme actually qualifies itself as a "Visual Odometry Centric" scheme: this in the sense that the STE and SQE modules, plus the associated additional sensors, have been adapted to the state space model describing the VO module. In the case we had instead started from IMU and/or WO sensor systems, the state space model most probably would have changed, becoming a discrete time, kinematic, state space model (possibly including the vehicle velocities as part of the state), instead of the two-step delay one resulting from the VO. Consequently, the eventual successive integration of a VO system should have been adapted to such a kinematic model, thus possibly leading to an integration scheme with different characteristics, worth to be investigated and compared to the developed one. 


\section{REFERENCES}

1. Nister, D., O. Naroditsky, and J. Bergen. 2006. Visual odometry for ground vehicle applications. J. Field Robotics 23(1):3-20.

2. Maimone, M., Y. Cheng, and L. Matthies. 2007. Two years of visual odometry on the Mars exploration rovers. J. Field Robotics 24(3):169-86.

3. Ducco, D., and F. Frassinelli. 2010. Analysis and realization of a visual odometry system. Master Thesis. University of Genoa.

4. Zereik, E., D. Ducco, F. Frassinelli, and G. Casalino. 2010. A study on planetary visual odometry optimization: Time constraints and reliability. 10th Symposium (International) on Artificial Intelligence, Robotics and Automation in Space.

5. Bar-Shalom, Y., X. Rong Li, and T. Kirubarajan. 2001. Estimation with application to tracking and navigation. John Wiley.

6. Doucet, A., N. Freitas, and N. Gordon. 2001. Sequential Monte Carlo methods in practice. Springer.

7. Ristic, B., S. Arulampalam, and N. Gordon. 2004. Beyond the Kalman filter: Particle filters for tracking applications. Artech House.

8. Thrun, S., W. Burgasrd, and D. Fox. 2005. Probabilistic robotics. The MIT Press. 